\section{RSP}

http://www.rsp.fsp.usp.br/
Revista de Saúde Pública

\title{
Políticas de equidad en los planes de salud: accesibilidad ¿y algo más?
}

\author{
Bran Barral Buceta' iD, Ramón Bouzas Lorenzo' iD, Andrés Cernadas Ramos' iD, \\ Ángela Fernández da Silva' iD \\ I Universidade de Santiago de Compostela. Facultad de Ciencias Políticas y Sociales. Departamento de Ciencia \\ Política y Sociología. Santiago de Compostela, Galicia, España
}

\section{RESUMEN}

OBJETIVO: Examinar el enfoque adoptado por los planes de salud de las comunidades autónomas de España verificando el peso otorgado al concepto de equidad; detectar a qué colectivos o situaciones se hace referencia; así como distinguir la perspectiva de abordaje del mismo, desde el acceso, la igualación o la equiparación.

MÉTODOS: Estudio cualitativo, de análisis de contenido mediante Nvivo12, realizado en 2020 sobre planes de salud en vigencia a fecha de 2019 en las diferentes regiones (comunidades autónomas) de España. Se han recopilado 16 planes de salud regionales vigentes para establecer categorías base (equidad, accesibilidad e igualdad) y determinar términos asociados a través de Nvivo12, a partir de los que se realizó un análisis de contenido.

RESULTADOS: El concepto de equidad no resulta destacado por los planes de salud autonómicos y su relevancia es superada por los conceptos de accesibilidad e igualdad. El empleo de estos tres conceptos está asociado a diversas categorías que connotan circunstancias, condiciones o colectivos a los que los planes prestan mayor atención.

Correspondencia:

Bran Barral Buceta

Universidade de Santiago de

Compostela

Facultad de Ciencias Políticas y Sociales

Av. Dr. Ángel Echeverri, s/n,

Campus sur.

Santiago de Compostela, Espanha.

CP 15782.

E-mail: bran.barral.buceta@usc.es

Recibido: 20 abr 2020

Aprobado: 1 set 2020

Cómo se cita: Barral B, BouzasLorenzo R, Cernadas A,

Fernández da Silva A. Políticas

de equidad en los planes de

salud: accesibilidad ¿y algo más?

Rev Saude Publica. 2021;55:31.

https://doi.org/10.11606/s1518-

8787.2021055002560

Copyright: Este es un artículo de el acceso abierto distribuido bajo la términos de la licencia Atribución Creative Commons, lo que permite el uso ilimitado, distribución y reproducción en cualquier medio, siempre que el autor y la fuente los originales se acreditan.
CONCLUSIONES: Los resultados obtenidos coinciden con estudios previos sobre los contenidos y orientación de los planes de salud, revelando una presencia discreta del concepto de equidad en los enfoques adoptados, sin que ello menoscabe el alineamiento de las políticas de salud respecto de las visiones emanadas de organizaciones transnacionales. Se detecta la existencia de un colectivo al que se presta especial atención desde el enfoque de accesibilidad, la población con diversidad funcional.

DESCRIPTORES: Planes de Sistemas de Salud. Equidad en el Acceso a los Servicios de Salud. Equidad en Salud. Estrategias para Cobertura Universal de Salud. Políticas Públicas de no Discriminación. Investigación Cualitativa. 


\section{INTRODUCCIÓN}

En las últimas décadas, la concepción de la salud y sus determinantes ha adoptado enfoques más proactivos y preventivos en consonancia con principios, entre ellos la defensa de la equidad, enfatizados por la Organización Mundial de la Salud (OMS) ${ }^{1-4}$.

Las interpretaciones del concepto de equidad son amplias ${ }^{5,6}$ y comprenden desde el alineamiento al antiintervencionismo estatal a la defensa de la igualdad de capacidades básicas y el máximo respaldo del Estado, pasando por la defensa de la equidad en el acceso ${ }^{6}$. Las primeras posturas se distancian de los principales debates sobre la equidad al asentarse en la premisa de que la desigualdad es inherente a la libertad de elección, si bien algunos autores optan por fijar un conjunto de servicios básicos ${ }^{7,8}$. Las demás alternativas, en mayor o menor medida defienden el diseño de políticas con la finalidad de atenuar o erradicar las inequidades en salud. Entre estas últimas, los estados tienen un papel más relevante" con propuestas orientadas principalmente a defender la universalidad-cuyo referente es el Beveridge Report ${ }^{10}$-, a proveer sistemas de seguridad social y a introducir los llamados means-testing programs ${ }^{11,12}$-una suerte de rentas de inserción social o discriminación positiva-, formas, todas ellas, de intervención no exentas de debate ${ }^{13-15}$.

La OMS ${ }^{(1-4)}$ se aproximó al concepto de "capacidades básicas" con base en las aportaciones de $\operatorname{Sen}^{16,17}$ y Nussbaum ${ }^{18,19}$, que acepta las responsabilidades personales en el estado de salud y defiende la actuación sobre cualquier factor causante de inequidad -condiciones socioeconómicas, sociodemográficas, geográficas, étnicas o de género desfavorables- que pueda introducir desigualdades de bienestar. De la propuesta deriva el concepto "desigualdad evitable”5 desarrollado por la OMS.

España, al igual que los otros países europeos y de la Organización para la Cooperación y el Desarrollo Económicos (OCDE), ha incluido el principio de equidad en las políticas de salud, acentuando su asimilación durante la primera década de este siglo y revisando su pervivencia en el contexto de la crisis financiera de $2008^{20-23}$.

Dejando al margen el tratamiento de la pertinencia de las diferentes aplicaciones de la equidad a la realidad social, este trabajo se interesa por el modo en que los servicios sanitarios de las Comunidades Autónomas ${ }^{a}$ (en adelante, CC.AA.) introducen la cuestión de la equidad y qué colectivos o circunstancias tienen en cuenta al abordarla. El objetivo es revelar la presencia del concepto de equidad en los planes de salud, identificar en ellos los principales temas asociados yel tratamiento que se les concede, contrastando sus orientaciones en el marco delas políticas públicas de salud con las visiones auspiciadas por el Ministerio de Sanidad, la Unión Europea (UE) y la OMS.

${ }^{a}$ Cabe recordar que, según la vigente Constitución de 1978, España es un Estado descentralizado territorialmente en 17 comunidades con amplias competencias sobre diversas materias y servicios entre ellos, los de salud. La Administración central se reserva las competencias sobre sanidad exterior y la coordinación del sistema sanitario en general, transfiriéndose el resto de competencias sobre los servicios de salud a las CC.AA. Dado el actual alto nivel de descentralización territorial, se podría afirmar que no hay un único sistema sanitario en el país, sino 17 subsistemas, pudiendo algunas comunidades ofrecer servicios diferentes, si bien en los aspectos básicos el sistema es relativamente homogéneo para el conjunto del Estado.

\section{MÉTODOS}

\section{Diseño}

Siguiendo las pautas del análisis comparativo constante ${ }^{24}$, mediante el examen de contenidos ${ }^{25-27}$ apoyado en una aplicación de soporte a la investigación cualitativa -Nvivol2-, se han revisado los planes de salud de las diferentes CC.AA.

\section{Muestra}

El estudio comprende los planes generales de salud vigentes -de nueva elaboración o prorrogados- promovidos por 16 CC.AA.

- Andalucía: IV Plan Andaluz de Salud (2013-2020)

- Aragón: Plan de Salud Aragón 2030

- Baleares: Plan Estratégico 2016-2020 
${ }^{\mathrm{b}}$ Cabe reseñar que estas tres categorías contradicen el criterio de "exclusión mutua", básico para la configuración de categorías, algo que el propio equipo de investigación pretendió evitar. Esta circunstancia forma parte de las excepciones que Bardin ${ }^{25}$ contempla para el fenómeno que denomina "multicodificación". Es decir, dada la imprecisión con que en ocasiones se hace referencia a ideas o ítems conceptualizados de forma diferente por la literatura, se pueden codificar estos elementos sin que necesariamente su codificación dentro de una categoría los excluya de su pertenencia a otra. Esto ocurre con las denominadas $\mathrm{CB}$, que son utilizadas en algunas ocasiones como sinónimos. Con la pretensión de lograr establecer si existen cuestiones específicas de cada una de ellas y, a su vez, encontrar aquellas otras que sean transversales a las tres, se ha optado en este caso por emplear esta opción, de forma que en los casos en que existan segmentos de texto que hagan una mención de 2 o incluso de $3 \mathrm{CB}$, se considere que los temas abordados sean parte de las tres categorías.

c Estas subcategorías responden a la configuración habitual de las categorías, siendo completamente excluyentes y encontrándose contenidas en alguna o todas las anteriores.
- Canarias: Plan de Salud de Canarias 2016-2017: Entre la crisis y la transformación para la innovación en la gestión de la salud de los servicios

- Cantabria: Plan de Salud de Cantabria 2014-2019

- Castilla-La Mancha: Estrategia 20-20 de Salud y Bienestar Social: El modelo de Castilla-La Mancha

- Castilla y León: IV Plan de Salud de Castilla y León (2020)

- Cataluña: Plan de salud de Cataluña 2016-2020

- Comunidad Valenciana: IV Plan de Salud 2016-2020 Comunitat Valenciana

- Extremadura: Plan de Salud de Extremadura 2013-2020

- Galicia: Estrategia Sergas 2020

- La Rioja: III Plan de Salud de La Rioja (2013-2020)

- Navarra: Plan de Salud de Navarra 2014-2020

- País Vasco: Plan de Salud Euskadi 2013-2020

- Principado de Asturias: Plan Sociosanitario del Principado de Asturias (2018-2021)

- Región de Murcia: Plan de Salud de 2010-2015 de la Región de Murcia

Dichos planes forman parte del estudio al cumplir los criterios de homogeneidad, representatividad, pertinencia y exhaustividad que propone Bardin ${ }^{25}$. Se buscaba analizar los planes generales sobre salud lo más actuales posibles. Es por ello que no se ha podido analizar ningún plan de la autonomía de Madrid dado que siguiendo la regla de la homogeneidad no se pudo encontrar un plan con estas características, siendo todos los recabados parciales y específicos.

\section{Análisis}

La literatura identifica factores o determinantes de la salud, que, por influencia de las instituciones transnacionales de referencia, era esperable encontrar reproducidos, en mayor o menor medida, en los planes de salud.

Sin embargo, el análisis de contenido desde la perspectiva de Bardin ${ }^{25}$, especialmente del enfoque emergente ${ }^{24}$ adoptado, obliga a extraer del objeto de estudio las categorizaciones y significados, y no a prefijarlos.

Así, durante la fase de preanálisis ${ }^{25}$, se procedió a la selección de los fragmentos de los planes en que se abordaban contenidos vinculados a la equidad. Desde esta primera aproximación, se detectaron varios ítems claves y se expandió la selección de fragmentos de texto -en consecuencia con la perspectiva emergente adoptada- a otros dos campos de significados que se sintetizan en los conceptos de "Accesibilidad" e "Igualdad", que se han denominado nodos o categorías base (en adelante, CB). El concepto "nodo" es propio de la terminología de la aplicación Nvivo y se refiere a una categoría que agrupa significantes similares: cada categoría opera como conjunto de semas relacionados con aquel que la nombrab ${ }^{b}$

Posteriormente se procedió al análisis de contenido y, como es propio de un enfoque de comparación constante, se conformaron nuevas subcategorías ${ }^{c}$ según la frecuencia de la aparición. Algunas de ellas ampliamente recogidas en la bibliografía consultada, y otras, menos comunes, derivadas del diseño adoptado. Como resultado, se fijaron las siguientes categorías clave (en adelante, CC): Calidad, Diversidad Funcional, Género, Grupos Sociales Vulnerables, Inmigración, Personas Mayores, Rural y Enfermedades Mentales. 
La diferencia entre las CB y CC radica en su función en el proceso de análisis: las "base" articulan la selección de los segmentos del plan analizados (operan como significantes marco); y las "clave" determinan el contenido dentro de esa selección. En otras palabras, las categorías base son los contenedores (los segmentos de texto englobados en el análisis) de lo que se habla o temas tratados (categorías clave). Este es otro de los motivos que ha llevado al equipo investigador a aceptar la "multicodificación" en estos tres casos, dado que permite realizar un análisis temático de las CC ajustado a cada una de las CB y, a partir del mismo, establecer las similitudes y diferencias entre los distintos paradigmas representados.

En el Cuadro 1 se recogen las operacionalizaciones realizadas para conformar las diversas categorías base y clave que se han mencionado en el artículo, así como los términos o lexemas que se incluyeron en el análisis ${ }^{\mathrm{d}}$.

\section{RESULTADOS}

En primer lugar, se ha determinado en qué medida los planes de salud contienen referencias a los conceptos asociados a la equidad en salud (Figura 1).

Tal y como se aprecia, el concepto más extensamente detectado dentro de los planes de salud es el de Accesibilidad, con un 2,06\% (de media), superior al tratamiento de las restantes CB en 11 de los planes autonómicos de salud analizados. Sin embargo, el concepto Igualdad alcanza mayores cotas de mención (en cuanto a máximos, siendo su media inferior con un $1,87 \%$ ), destacando en el 6,53\% de los contenidos del plan de salud de Andalucía, el 6,12\% del plan del País Vasco y el 4,51\% en el caso de Aragón.

A su vez, el concepto Equidad tiene una mayor relevancia en el plan de salud del País Vasco ( $4,23 \%$ de contenidos) y, en menor medida, en el plan autonómico andaluz ( $1,77 \%$ y en el de las Islas Canarias (1,67\%), obteniendo una media de codificación del 1,08\%, la menor de las tres CB.

Por su parte, el concepto Accesibilidad alcanza su presencia más elevada en el plan autonómico de Castilla-La Mancha (3,92\%), seguido por el del País Vasco (3,33\%) y el de Asturias (2,84\%).

Resulta, asimismo, llamativa la ausencia de contenidos hallada en algunos planes: el tratamiento del concepto Igualdad es exiguo -menos del 0,25\% - en los planes de Asturias, Islas Baleares y La Rioja; el concepto Equidad es mínimamente abordado -menos del 0,7\%- en los planes de Islas Baleares, Cantabria y Castilla-La Mancha; y el término Accesibilidad alcanza sus cifras más bajas -menos del 1,2\% - en los planes de Islas Baleares, Murcia y Extremadura.

Cuadro 1. Relación de categorías y de los términos o lexemas aplicados y englobados en la codificación.

${ }^{\mathrm{d}}$ Fruto del propio análisis de la frecuencia de los lexemas del contenido de los planes y gracias a la herramienta del programa Nvivo que permite dicho criterio de búsqueda por lexema, amparados así mismo por la ampliación a través de los principales diccionarios de sinónimos y la competencia lingüística nativa del equipo investigador.

\begin{tabular}{|c|c|c|}
\hline Categoría & & Términos o lexemas empleados \\
\hline \multirow{3}{*}{$\begin{array}{l}\text { Categorías } \\
\text { Base }\end{array}$} & Equidad & Equidad, Equitativo*, Inequitativ* \\
\hline & Igualdad & Igualdad, Desigualdad*, Igualitar* \\
\hline & Accesibilidad & Accesibilidad, Accesible, Acceso, Inaccesible, Inaccesibilidad \\
\hline \multirow{8}{*}{$\begin{array}{l}\text { Categorías } \\
\text { Clave }\end{array}$} & $\begin{array}{l}\text { Diversidad } \\
\text { funcional }\end{array}$ & $\begin{array}{c}\text { Diversidad funcional, Discapacitad*, Discapacidad, Dependient*, } \\
\text { Dependencia, Independencia }\end{array}$ \\
\hline & Rural & $\begin{array}{l}\text { Rural, Periferia, Núcleos poblacionales pequeños/alejados, Dispersos, } \\
\text { Dispersión, Difícil acceso, Alejad*, Zona*, Geografía*, Geográfic* }\end{array}$ \\
\hline & $\begin{array}{l}\text { Grupos sociales } \\
\text { vulnerables }\end{array}$ & $\begin{array}{c}\text { Grupo social, Grupos sociales, Colectivos en situación de riesgo, } \\
\text { Vulnerabilidad, Vulnerabl*, Desfavorecid*, Colectivo* }\end{array}$ \\
\hline & Género & Género, Hombre*, Mujer*, Violencia, Maltrato, Machismo \\
\hline & Inmigración & Inmigración, Inmigrante, Raza, Etnia, Étnic*, Lengua, Habla, Extranjer* \\
\hline & Personas Mayores & Mayor*, Tercera Edad/3 Edad, envejecer, envejecimiento, ancian* \\
\hline & Calidad & Calidad \\
\hline & Mental & Mental* \\
\hline
\end{tabular}

* Se han seleccionado aquellas palabras que empiezan de ese modo, de forma que se pueden usar para obviar el género o los plurales de las mismas. Se trata de una práctica propia del programa de Nvivo y de sus criterios de búsqueda, por ello se plasma de esa manera. Estos términos se han revisado caso a caso para observar la pertinencia de los mismos, descartando los que no tenían un significado coherente con lo buscado. 


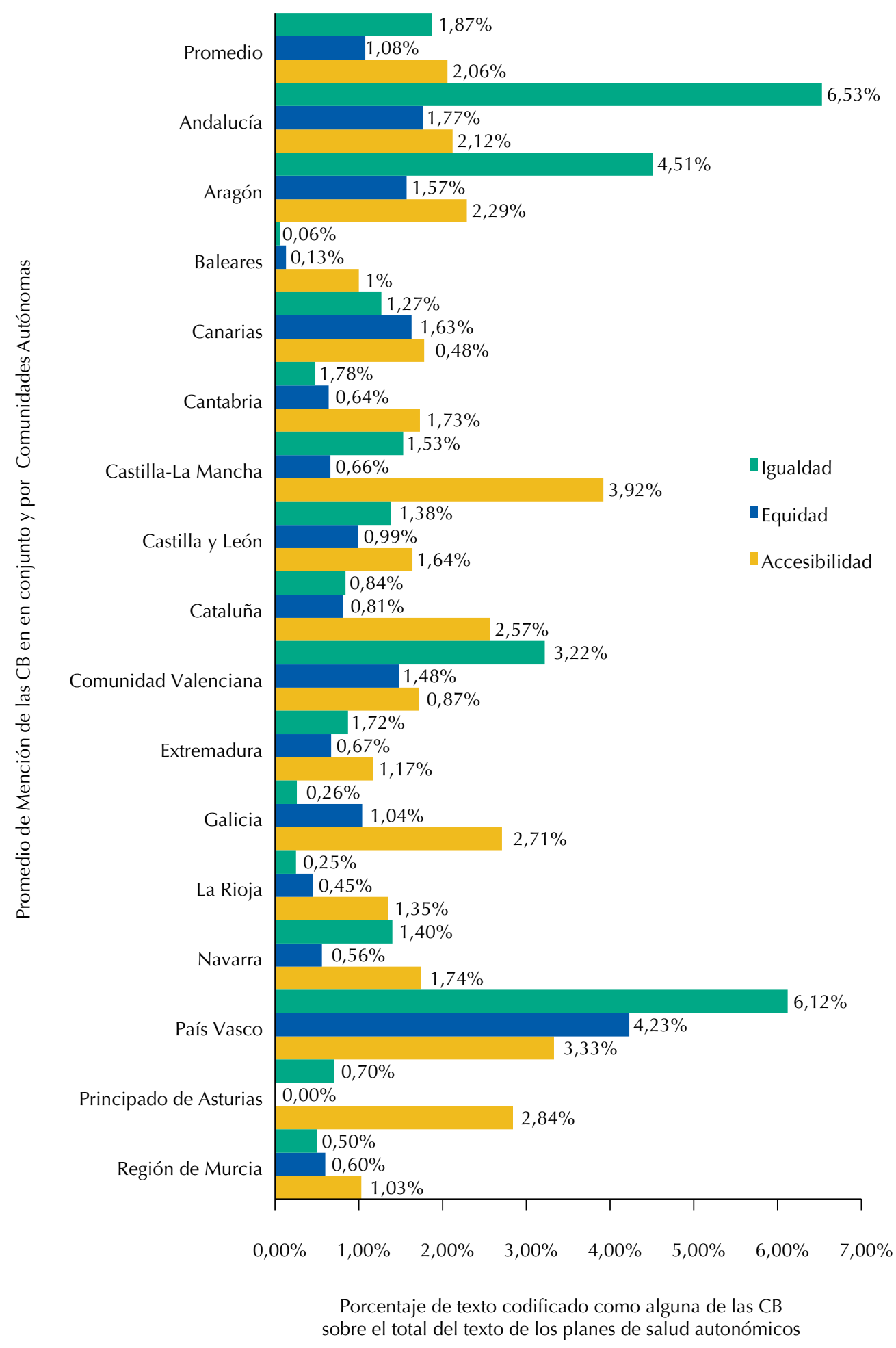

Figura 1. Porcentaje de texto codificado según las categorías base sobre el total del texto recogido en los planes autonómicos.

Pero ¿a qué se refiere cuando se habla de equidad, igualdad y accesibilidad?

Aunque, como se señaló, los temas asociados a las CB -Equidad, Igualdad y Accesibilidadson incorporados a la práctica en totalidad de los planes de salud, el tratamiento de CC encuentra diferencias significativas, según muestra la distribución de menciones en los planes de salud autonómicos (Tabla 1).

Desde el punto de vista de la diversidad temática abarcada, la menor variedad de CC se detecta en los planes de Galicia y Baleares -en Galicia solo se trata el concepto Calidad y 
Tabla 1. Menciones a las categorías base y claves en los planes de salud autonómicos por frecuencia de codificación (sobre total codificado por categoría).

\begin{tabular}{|c|c|c|c|c|c|c|c|c|c|c|c|c|}
\hline & Accesibilidad & Calidad & $\begin{array}{l}\text { Diversidad } \\
\text { funcional }\end{array}$ & Equidad & Género & $\begin{array}{c}\text { Grupos } \\
\text { Sociales } \\
\text { Vulnerables }\end{array}$ & Igualdad & Inmigración & $\begin{array}{l}\text { Personas } \\
\text { Mayores }\end{array}$ & $\begin{array}{c}\text { Enfermedades } \\
\text { Mentales }\end{array}$ & Rural & $\begin{array}{c}\text { Media de } \\
\text { menciones } \\
\text { sobre total } \\
\text { por CC. } \\
\text { AA. }\end{array}$ \\
\hline Andalucía & $5,38 \%$ & $10,86 \%$ & $4,34 \%$ & $8,01 \%$ & $7,61 \%$ & $5 \%$ & $16,90 \%$ & $5,36 \%$ & $0,67 \%$ & $6,52 \%$ & $10,10 \%$ & $7,34 \%$ \\
\hline Aragón & $3,27 \%$ & $1,33 \%$ & $0,91 \%$ & $4,14 \%$ & $0,74 \%$ & $3,50 \%$ & $7,99 \%$ & $5,85 \%$ & $4,67 \%$ & $2,10 \%$ & $8,75 \%$ & $3,93 \%$ \\
\hline Baleares & $2,16 \%$ & $0,27 \%$ & $0,16 \%$ & $0,54 \%$ & $0 \%$ & $0 \%$ & $0,17 \%$ & $1,17 \%$ & $0 \%$ & $0 \%$ & $0 \%$ & $0,41 \%$ \\
\hline Canarias & $6,13 \%$ & $6,63 \%$ & $0,99 \%$ & $10,14 \%$ & $0,34 \%$ & $3,88 \%$ & $5,24 \%$ & $1,17 \%$ & $0 \%$ & $2,05 \%$ & $3,52 \%$ & $3,64 \%$ \\
\hline Cantabria & $16,56 \%$ & $3,71 \%$ & $18,85 \%$ & $11,04 \%$ & $3,69 \%$ & $8,13 \%$ & $5,52 \%$ & $10,62 \%$ & $5,71 \%$ & $52,70 \%$ & $5,44 \%$ & $12,91 \%$ \\
\hline $\begin{array}{l}\text { Castilla-La } \\
\text { Mancha }\end{array}$ & $2,78 \%$ & $0,80 \%$ & $3,28 \%$ & $0,81 \%$ & $0,11 \%$ & $0,48 \%$ & $1,25 \%$ & $0,39 \%$ & $0,67 \%$ & $0 \%$ & $3,47 \%$ & $1,28 \%$ \\
\hline $\begin{array}{l}\text { Castilla y } \\
\text { León }\end{array}$ & $6,93 \%$ & $2,12 \%$ & $7,18 \%$ & $7,51 \%$ & $2,61 \%$ & $1,67 \%$ & $7,10 \%$ & $4,42 \%$ & $0,46 \%$ & $6,52 \%$ & $9,74 \%$ & $5,11 \%$ \\
\hline Cataluña & $5,99 \%$ & $42,71 \%$ & $1,53 \%$ & $3,68 \%$ & $58,55 \%$ & $19,82 \%$ & $2,65 \%$ & $0 \%$ & $59,18 \%$ & $2,09 \%$ & $0 \%$ & $17,84 \%$ \\
\hline $\begin{array}{l}\text { Comunidad } \\
\text { Valenciana }\end{array}$ & $8,41 \%$ & $3,98 \%$ & $4,13 \%$ & $12,43 \%$ & $3,98 \%$ & $21,28 \%$ & $17,63 \%$ & $16,17 \%$ & $0,64 \%$ & $0 \%$ & $0 \%$ & $8,06 \%$ \\
\hline Extremadura & $10,98 \%$ & $7,43 \%$ & $13,31 \%$ & $11,92 \%$ & $10,34 \%$ & $5,88 \%$ & $10,23 \%$ & $9,97 \%$ & $17,08 \%$ & $2,17 \%$ & $47,28 \%$ & $13,33 \%$ \\
\hline Galicia & $1,61 \%$ & $1,06 \%$ & $0 \%$ & $1,17 \%$ & $0 \%$ & $0 \%$ & $0,18 \%$ & $0 \%$ & $0 \%$ & $0 \%$ & $0 \%$ & $0,37 \%$ \\
\hline La Rioja & $10,97 \%$ & $5,04 \%$ & $22,17 \%$ & $6,91 \%$ & $6,59 \%$ & $7,55 \%$ & $2,55 \%$ & $30,04 \%$ & $6,26 \%$ & $8,70 \%$ & $6,01 \%$ & $10,25 \%$ \\
\hline Navarra & $6,42 \%$ & $3,45 \%$ & $9,32 \%$ & $3,86 \%$ & $0,68 \%$ & $7,27 \%$ & $6,08 \%$ & $7,60 \%$ & $0,96 \%$ & $2,11 \%$ & $3,11 \%$ & $4,62 \%$ \\
\hline País Vasco & $6,39 \%$ & $8,22 \%$ & $1,94 \%$ & $14,58 \%$ & $4,03 \%$ & $7,65 \%$ & $13,86 \%$ & $0,81 \%$ & $2,03 \%$ & $1,98 \%$ & $0,62 \%$ & $5,65 \%$ \\
\hline $\begin{array}{l}\text { Principado } \\
\text { de Asturias }\end{array}$ & $3,02 \%$ & $1,59 \%$ & $6,86 \%$ & $0 \%$ & $0,45 \%$ & $4,37 \%$ & $0,91 \%$ & $0,39 \%$ & $1,67 \%$ & $10,87 \%$ & $1,29 \%$ & $2,86 \%$ \\
\hline $\begin{array}{l}\text { Región de } \\
\text { Murcia }\end{array}$ & $3 \%$ & $0,80 \%$ & $5,03 \%$ & $3,26 \%$ & $0,28 \%$ & $3,52 \%$ & $1,74 \%$ & $6,04 \%$ & $0 \%$ & $2,19 \%$ & $0,67 \%$ & $2,41 \%$ \\
\hline $\begin{array}{l}\text { Total } \\
\text { Menciones } \\
\text { a la } \\
\text { categoría }\end{array}$ & $100,00 \%$ & $100,00 \%$ & $100,00 \%$ & $100,00 \%$ & $100,00 \%$ & $100,00 \%$ & $100,00 \%$ & $100,00 \%$ & $100,00 \%$ & $100,00 \%$ & $100,00 \%$ & $100,00 \%$ \\
\hline
\end{tabular}

Nota: Los colores atienden a la intensidad y dirección de la codificación; aquellos más próximos al rojo denotan menor porcentaje de mención; los verdes mayor; y los tonos amarillos los más moderados. En este caso representa los valores en columna y responde, por lo tanto, al porcentaje de segmentos del texto que se han codificado en cada categoría según el total individual de cada una de ellas. Como resultado, se muestra qué comunidades enfatizan en mayor o menor medida cada tema.

En las columnas que se hace mención al número de páginas de los planes, se crea un indicador en la última columna que atiende al ratio de codificación media por página del plan, que permite relacionar el porcentaje de codificación de cada comunidad con su número de páginas: cuanto más verde mayor es el ratio entre páginas y codificación y, por ende, mayor la atención prestada a la equidad; y los más próximos al rojo figuran las comunidades que menor atención prestan.

en Islas Baleares, Inmigración y Calidad- mientras que son varios los planes autonómicos de salud (Andalucía, Aragón, Cantabria, Castilla y León, Extremadura, La Rioja y Navarra) que comprenden la totalidad de CC, si bien con desigual atención.

A su vez, respecto a la intensidad de tratamiento temático, destaca el plan de Cataluña, que concentra más de la mitad de las menciones que a tres CC se hacen en los planes autonómicos: Personas Mayores (59,18\%), Género (58,55\%) y Calidad (42,71\%). Otros dos planes autonómicos reúnen la mayor parte de referencias a dos CC: Enfermedades Mentales (52,7\%), en el caso de Cantabria, y Rural (47,28\%), en de Extremadura.

Con menor intensidad, otras CC en las que despuntan los planes de salud son: Inmigración (La Rioja, 30,04\%), Grupos Sociales Vulnerables (Cataluña, 21,28\%; Comunidad Valenciana, 19,82\%) y Diversidad Funcional (La Rioja, 22,17\%; Cantabria, 18,85\%).

Por otra parte, es importante situar el tratamiento de los temas en el contexto de la relevancia que tienen en los planes, ya que no necesariamente el que un plan acumule el mayor número de menciones a una categoría implica que a esta se le conceda mayor importancia respecto a las demás, como se podrá ver a continuación.

En primer lugar, cabe destacar que las CB poseen mayor presencia, algo lógico dada la selección del texto en función de las mismas. En este sentido, la CB que más codificación acumula de media 
es Accesibilidad, con un 36,09\% de las menciones; seguida por Igualdad, un 22,77\%; y Equidad, 16,24\%. Esta última mantiene un porcentaje de menciones similar al alcanzado por la CCDiversidad Funcional (13,72\%), uno de los temas más importantes de los fragmentos seleccionados.

Las restantes CC se ven subsumidas en cuanto a número de menciones: Grupos Sociales Vulnerables (3,77\%), Personas Mayores (2,42\%), Inmigración (2,19\%), Género (1,17\%), Rural (1,31\%), Calidad (0,30\%) y Enfermedades Mentales (0,03\%).

Ponderando el peso de las $\mathrm{CC}$ al margen de las $\mathrm{CB}$ se facilita un análisis temático más afinado por comunidad (Tabla 2). Así, se observa que la categoría Diversidad Funcional destaca rotundamente sobre las demás (48,36\% de media, con solo Aragón, Cataluña y Galicia por debajo del $30 \%$ de menciones) y hay que descender hasta el 16,56\% de las codificaciones medias para encontrar la siguiente CC, Grupos Sociales Vulnerables - -destacan las menciones a este issue en los casos de la Comunidad Valenciana, País Vasco, Canarias, Cataluña y Aragón, todas por encima de un $20 \%$ de la codificación temática realizada mediante las CC-, o hasta el 11,40\% para encontrar la tercera categoría, Inmigración, que destaca su peso para las Islas Baleares con un 55,37\% y en menor medida Aragón y la Comunidad Valenciana con cifras en torno al $20 \%$.

La supremacía del concepto Diversidad Funcional está presente en la mayoría de las CC. AA., siendo uno de los targets más destacados -resulta especialmente visible en los planes de Castilla-La Mancha, Asturias, Cantabria, Castilla y León, Región de Murcia, La Rioja y Navarra-. Queda al margen Galicia, que aborda de manera exclusiva la equidad en salud relacionándola con la Calidad.

Tabla 2. Porcentaje de codificación de los issues abordados por las CC (sobre total codificado por planes de salud autonómicos).

\begin{tabular}{|c|c|c|c|c|c|c|c|c|c|}
\hline & Calidad & $\begin{array}{l}\text { Diversidad } \\
\text { funcional }\end{array}$ & Género & $\begin{array}{c}\text { Grupos } \\
\text { Sociales } \\
\text { Vulnerables }\end{array}$ & Inmigración & $\begin{array}{l}\text { Personas } \\
\text { Mayores }\end{array}$ & $\begin{array}{c}\text { Enfermedades } \\
\text { Mentales }\end{array}$ & Rural & Total \\
\hline Andalucía & $2,62 \%$ & $47,76 \%$ & $8,55 \%$ & $16,54 \%$ & $10,48 \%$ & $1,45 \%$ & $0,21 \%$ & $12,41 \%$ & $100,00 \%$ \\
\hline Aragón & $0,58 \%$ & $18,08 \%$ & $1,48 \%$ & $20,98 \%$ & $20,72 \%$ & $18,53 \%$ & $0,13 \%$ & $19,50 \%$ & $100,00 \%$ \\
\hline Baleares & $1,47 \%$ & $43,16 \%$ & $0,00 \%$ & $0,00 \%$ & $55,37 \%$ & $0,00 \%$ & $0,00 \%$ & $0,00 \%$ & $100,00 \%$ \\
\hline Canarias & $4,88 \%$ & $33,77 \%$ & $1,19 \%$ & $39,58 \%$ & $7,12 \%$ & $0,00 \%$ & $0,13 \%$ & $13,32 \%$ & $100,00 \%$ \\
\hline Cantabria & $0,30 \%$ & $73,85 \%$ & $1,48 \%$ & $9,57 \%$ & $7,41 \%$ & $4,45 \%$ & $0,54 \%$ & $2,39 \%$ & $100,00 \%$ \\
\hline $\begin{array}{l}\text { Castilla- La } \\
\text { Mancha }\end{array}$ & $0,43 \%$ & $81,14 \%$ & $0,30 \%$ & $3,58 \%$ & $1,71 \%$ & $3,28 \%$ & $0,00 \%$ & $9,56 \%$ & $100,00 \%$ \\
\hline Castilla y León & $0,48 \%$ & $71,93 \%$ & $2,69 \%$ & $5,04 \%$ & $7,87 \%$ & $0,91 \%$ & $0,19 \%$ & $10,89 \%$ & $100,00 \%$ \\
\hline Cataluña & $3,55 \%$ & $5,83 \%$ & $22,77 \%$ & $22,77 \%$ & $0,00 \%$ & $45,07 \%$ & $0,02 \%$ & $0,00 \%$ & $100,00 \%$ \\
\hline $\begin{array}{l}\text { Comunidad } \\
\text { Valenciana }\end{array}$ & $0,64 \%$ & $29,42 \%$ & $2,89 \%$ & $45,61 \%$ & $20,52 \%$ & $0,92 \%$ & $0,00 \%$ & $0,00 \%$ & $100,00 \%$ \\
\hline Extremadura & $0,60 \%$ & $49,75 \%$ & $3,94 \%$ & $6,60 \%$ & $6,63 \%$ & $12,73 \%$ & $0,03 \%$ & $19,71 \%$ & $100,00 \%$ \\
\hline Galicia & $100,00 \%$ & $0,00 \%$ & $0,00 \%$ & $0,00 \%$ & $0,00 \%$ & $0,00 \%$ & $0,00 \%$ & $0,00 \%$ & $100,00 \%$ \\
\hline La Rioja & $0,34 \%$ & $68,18 \%$ & $2,07 \%$ & $7,00 \%$ & $16,45 \%$ & $3,83 \%$ & $0,06 \%$ & $2,07 \%$ & $100,00 \%$ \\
\hline Navarra & $0,55 \%$ & $68,81 \%$ & $0,52 \%$ & $16,15 \%$ & $9,99 \%$ & $1,39 \%$ & $0,03 \%$ & $2,56 \%$ & $100,00 \%$ \\
\hline País Vasco & $3,26 \%$ & $35,43 \%$ & $7,58 \%$ & $42,19 \%$ & $2,68 \%$ & $7,46 \%$ & $0,12 \%$ & $1,28 \%$ & $100,00 \%$ \\
\hline $\begin{array}{l}\text { Principado de } \\
\text { Asturias }\end{array}$ & $0,39 \%$ & $77,62 \%$ & $0,53 \%$ & $14,93 \%$ & $0,78 \%$ & $3,79 \%$ & $0,33 \%$ & $1,63 \%$ & $100,00 \%$ \\
\hline $\begin{array}{l}\text { Región de } \\
\text { Murcia }\end{array}$ & $0,23 \%$ & $69,01 \%$ & $0,41 \%$ & $14,51 \%$ & $14,74 \%$ & $0,00 \%$ & $0,09 \%$ & $1,02 \%$ & $100,00 \%$ \\
\hline $\begin{array}{l}\text { Media de } \\
\text { mención a las } \\
\text { CB por CC. } \\
\text { AA. }\end{array}$ & $7,52 \%$ & $48,36 \%$ & $3,52 \%$ & $16,56 \%$ & $11,40 \%$ & $6,49 \%$ & $0,12 \%$ & $6,02 \%$ & $100,00 \%$ \\
\hline
\end{tabular}

Nota: Para una mayor claridad del peso de los contenidos dentro de cada plan, se pormenoriza en la frecuencia de mención relativa de cada una de las CC y se analiza el peso específico de cada una de ellas sobre las restantes (excluyendo, como se ha comentado, las CB). Así se destacan aquellos temas $\mathrm{o}$ issues abordados en mayor o menor medida por cada comunidad. El uso de colores sigue el patrón anteriormente aplicado, pero en este caso responde a las cifras ubicadas en las filas, por lo que muestra la importancia relativa de la CC en cada uno de los planes de forma individualizada y, por ende, en cada comunidad. 
La centralidad de la Diversidad Funcional asociada a la categoría Accesibilidad puede explicarse a partir de las medidas expuestas en los planes de salud y en cómo se argumentan sus causas: desde el acceso a las propias infraestructuras sanitarias hasta la adaptación de las páginas web a formatos más accesibles para los diferentes tipos de usuarios, pasando por el establecimiento de atenciones domiciliarias que faciliten la prestación de la atención sanitaria en condiciones de igualdad.

Las categorías Diversidad Funcional, Grupos Sociales Vulnerables e Inmigración centran la atención en la mayoría de contenidos que expresan la necesidad de potenciar la equidad en salud, articulando políticas o medidas que procuran mejorar la situación en que se encuentran estos colectivos.

En ese sentido, teniendo en cuenta que los campos semánticos de cada categoría implican no solo la consideración de una condición o unas circunstancias, sino que aluden a la porción de población por estas afectada, cabe destacar que entre los planes examinados también resultan principales las acciones en salud encaminadas a mejorar la situación de otros colectivos: Cataluña respecto a la CC Personas Mayores (45,07\%) y Género (22,77\%); o Extremadura y Aragón, al tratar la CC Rural (19,71\% y 19,50\%, respectivamente).

A partir de todos los datos anteriores, se ha representado (Figura 2) la situación de cada plan de salud según la relevancia concedida a cada CC.

A partir de esta figura se observa que hay varias CC. AA. cuya aproximación a la equidad se concentra en pocas CC, mientras otras abordan más targets y, por tanto, entienden que es una cuestión más amplia. En el primer grupo figuran Baleares, La Rioja, Navarra, Región de Murcia, Castilla y León, Cantabria, Asturias, Castilla-La Mancha y, finalmente, Galicia; en el segundo, Aragón, Andalucía, Canarias, Cataluña, Extremadura, País Vasco y la Comunidad Valenciana, todas ellas por debajo de la media de desviación típica y, por tanto, que tienden a observar y tener en cuenta más colectivos que las restantes.

\begin{tabular}{|c|c|c|c|}
\hline $\begin{array}{c}\text { Comunidades } \\
\text { Autónomas }\end{array}$ & $\begin{array}{c}\text { Desv. Típ } \\
\text { sobre el \%de } \\
\text { codificación de } \\
\text { cada plan a las }\end{array}$ & $\begin{array}{c}\text { Mediaa } \\
\text { rakking } \\
\text { en CC }\end{array}$ & $\begin{array}{c}\text { Posición } \\
\text { Ranking } \\
\text { medio) }\end{array}$ \\
\hline Aragón & 0,10 & 6 & 2 \\
\hline Andalucía & 0,17 & 5,5 & 1 \\
\hline Cataluña & 0,17 & 8 & 8 \\
\hline Canarias & 0,17 & 7,25 & 4 \\
\hline País Vasco & 0,17 & 6,75 & 3 \\
\hline Extremadura & 0,17 & 7,25 & 5 \\
\hline Comunidad Valenciana & 0,18 & 8,25 & 9 \\
\hline Baleares & 0,24 & 10,75 & 15 \\
\hline La Rioja & 0,25 & 8,375 & 10 \\
\hline Navarra & 0,25 & 8,625 & 12 \\
\hline Región de Murcia & 0,25 & 10,125 & 14 \\
\hline Castilla y León & 0,26 & 7,875 & 7 \\
\hline Cantabria & 0,27 & 7,5 & 6 \\
\hline Principado de Asturias & 0,29 & 8,375 & 11 \\
\hline Castilla-La Mancha & 0,30 & 10,125 & 13 \\
\hline Galicia & 0,38 & 12,625 & 16 \\
\hline
\end{tabular}

DESVIACIÓN TÍPICA DE LA PRESENCIA DE CODIFICACIÓN DE CC EN LOS PLANES DE SALUD

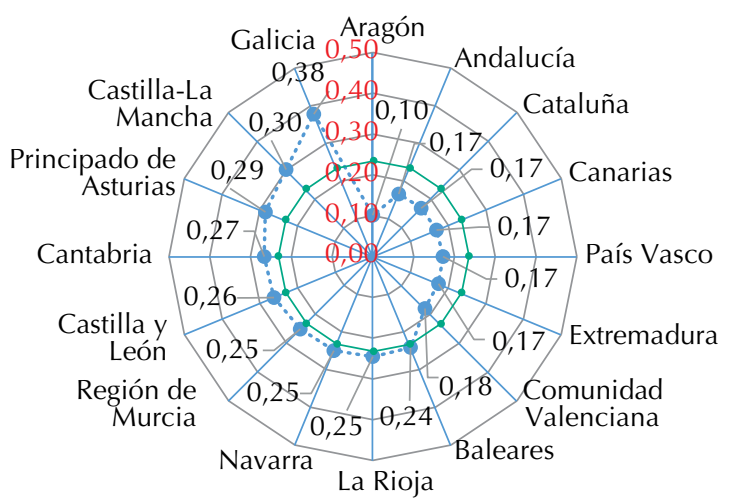

....... Desv. Típ sobre el \% de codificación de cada plan a las CC

Promedio de Desv.Típ de todas las CC para todas las CC. AA.

Nota: En la primera columna ("Desv. Típ. sobre el \% de codificación de cada plan a las CC"), los colores más cercanos al rojo indican que las comunidades se han centrado sobre todo en uno o pocos issues (CC); los más verdes, si han adoptado posturas respecto a la equidad que incluyen a multiplicidad de colectivos; y los tonos amarillos destacan las puntuaciones intermedias. Las siguientes dos columnas clasifican a las comunidades según la importancia relativa concedida a cada CC y a otorgarles un valor según la posición (de 1 a 16) que ocupan, obteniendo las medias, que, a su vez, en la tercera columna se pasan a un formato de ranking, ordenando las comunidades según su puntuación obtenida. Siguiendo la lógica de uso precedente, los colores son nuevamente representativos de la mayor o menor atención a pocos o múltiples CC: cuantas más CC se contemplen y se aborden en los planes (con cierta relevancia porcentual), menor será la media del ranking y a su vez la posición, lo que desde la perspectiva de esta investigación (de acuerdo con la perspectiva de la OMS de la existencia de un problema multicausal) resulta más adecuado para abordar esta problemática.

Figura 2. Ranking de las CCAA según la atención que prestan a las distintas CC en sus planes. 


\section{DISCUSIÓN}

Conocer cuál es la aproximación de cada comunidad a la equidad, en qué colectivos están centrando sus políticas sanitarias y con qué orientación, implica comprobar qué cuestiones no se están abordando, lo que plantea la pregunta sobre dicha ausencia teniendo en cuenta la consideración que actores transnacionales como la OMS y la propia UE poseen de la teoría de las capacidades básicas propuesta por Sen Se, $^{3,16,17,21}$.

Este estudio cualitativo corrobora los hallazgos de investigaciones precedentes, de que los planes de salud autonómicos contienen escasas referencias explícitas al concepto de equidad en salud ${ }^{20-23,28}$. Los planes focalizan su atención al abordaje de enfermedades concretas, la incorporación de tecnologías sanitarias y la reorganización de servicios, dejando en un segundo plano la cuestión de la equidad, que solo se revela, indirectamente, por medio de la demanda de una mejora del acceso a los servicios sanitarios, adoptando, en suma, una visión orientada a la perspectiva del acceso en igualdad de condiciones y no acorde con la propuesta de Sen y la OMS sobre la igualdad de capacidades y de la atención a los determinantes sociales.

Además, este acceso no se considera por todas las CC.AA. como un factor general y multicausal con diversos colectivos o issues clave a tener en cuenta, sino que se significan colectivos concretos, entre los que destaca el de las personas con Diversidad Funcional, principal target al que se dirigen las políticas de equidad en salud. En un segundo plano quedan las referencias a otros colectivos destacados como los Grupos Sociales Vulnerables y Personas Inmigrantes. Lo anterior no impide resaltar que el análisis confirma que los planes de salud vinculan la categoría Grupos Sociales Vulnerables a situaciones de discriminación económica, relación puesta en evidencia por organismos transnacionales como la OMS como fuente de inequidad asociada a pobreza y empeoramiento de la salud $\mathrm{d}^{5}$.

Por su parte, la atención a temas como género, calidad, personas mayores, ámbito rural o enfermedades mentales no se contempla de forma uniforme en los planes autonómicos. Sin embargo, este hecho no los convierte en cuestiones irrelevantes, al contrario, su ausencia evidencia la carente perspectiva multidimensional de las inequidades que existen en materia de salud. Todo esto tiene una grave repercusión, ya que puede comportar un déficit de atención a dichos colectivos, que padecen dificultades añadidas para poder ser usuarios de pleno derecho del servicio sanitario ${ }^{20-23,28,29}$.

Desde el punto de vista del promotor de los planes de salud -las Administraciones Autonómicas- se pueden distinguir, por una parte, comunidades que abordan la equidad como una cuestión muy focalizada en uno o dos colectivos y las que tratan el problema de una manera más comprehensiva, más global y próxima al concepto de capacidades básicas, si bien ninguna de ellas va más allá del factor accesibilidad y no tienen en cuenta completamente los demás componentes de la inequidad en salud como defienden la OMS y la literatura sobre el caso español ${ }^{20-23,28-30}$.

Aunque la concepción del diseño de las políticas de salud pueda adolecer de insuficiente atención específica a determinados colectivos, la implementación los debería tener en cuenta y evitar, así, graves inequidades en los niveles de uso del servicio: el acceso a un mismo servicio no garantiza una igual atención si, por ejemplo, algunos usuarios carecen de recursos económicos para sufragar su medicación, tienen dificultades para entender el idioma en que se les atiende o son incapaces de visualizar una prescripción. La calidad de su atención, incluso la misma atención, está en riesgo ${ }^{6,16,17,19}$.

Todas las categorías clave que se han detectado son perfectamente aplicables al conjunto de las CC. AA. No resaltarlas no deriva de la inexistencia de colectivos concretos, sino de una elección política de los target de los planes. 
Confirmando lo establecido en investigaciones previas que apuntaban en la misma dirección ${ }^{20-23,28-30}$, los resultados certifican la falta de una mayor plasmación de la equidad en los planes y de en general abordar pocos de los temas clave. Depender de que solo la implementación cubra las carencias de la planificación y, como sucedería en este caso, pueda mitigar un exiguo tratamiento de la equidad, no resulta una práctica solvente.

Este último, el de la implementación, es un terreno situado más allá de los propósitos de este trabajo, y será en el estudio de la implementación de lo que los planes de salud proponen en que realmente se pueda constatar cómo y en qué se traducen las ideas de partida ${ }^{20,31}$ que han sido objeto de este análisis. En este sentido, cabrá esperar que esta investigación estimule nuevos estudios al respecto y sirva como marco que permita análisis más profundos de los planes de salud vigentes y venideros.

\section{REFERENCIAS}

1. World Health Organization. Health21: the health for all policy framework for the WHO European Region. Copenhagen (DK): WHO Regional Office for Europe; 1999 [citado 23 set 2019]. (European Health for All Series; n 6. Disponible en: http://www.euro.who.int/_data/ assets/pdf_file/0010/98398/wa540ga199heeng.pdf

2. Marmot M; Commission on Social Determinants of Health. Achieving health equity: from root causes to fair outcomes. Lancet. 2007;370(9593):1153-63. https://doi.org/10.1016/S01406736(07)61385-3

3. Dahlgren G, Whitehead M. Policies and strategies to promote social equity in health; background document to WHO - Strategy paper for Europe. Stockholm (SE): Institute for Future Studies; 1991 [citado 24 set 2019]. Disponible en: https://core.ac.uk/download/pdf/6472456.pdf

4. World Health Organization. Working towards achieving the Sustainable Development Goals: a WHO toolkit. Geneva (CH): WHO; 2018 [citado 24 set 2019]. Disponible en: https://apps.who. int/iris/handle/10665/274261

5. Whitehead M. The concepts and principles of equity and health. Int J Health Serv.1992;22(3):429-45. https://doi.org/10.2190/986L-LHQ6-2VTE-YRRN

6. Cernadas Ramos A. La salud y el acceso a los sistemas sanitarios públicos: desigualdades e inequidades. Madrid: Editorial Síntesis; 2010.

7. Enthoven AC. Is consumer choice and competition in health care the wave of the future? Hosp Financ Manage. 1980;34(11):12-5, 18, 20 passim.

8. Nozick R. Anarquía, Estado y utopía. [S.I.]: Editorial Innisfree; 2014.

9. Navarro V, Quiroga A. Políticas de estado de bienestar para la equidad. Gac Sanit. 2004;18(4):147-57.

10. Beveridge WH. Social insurance and allied services: report by Sir William Beveridge. Bull World Health Organ. 2000;78(6):847-55. 2000 [citado 27 set 2019];78(6):847-55. Disponible en: https://apps.who.int/iris/handle/10665/57560

11. Korpi W, Palme J. The paradox of redistribution and strategies of equality: welfare state institutions, inequality, and poverty in the western countries. Am Sociol Rev. 1998;63(5):661-87. https://doi.org/10.2307/2657333

12. Diderichsen F. Income maintenance policies: determining their potential impact on socioeconomic inequalities in health. In: Mackenbach J, Bakker M, editors. Reducing inequalities in health: a European perspective. London (UK): Routledge; 2002. p. 53-66.

13. Sojo A. Reformas de gestión en salud en América Latina: los cuasimercados de Colombia, Argentina, Chile y Costa Rica. Santiago de Chile: Editorial Cepal; 2000.

14. Altman D, Frist WH. Medicare and Medicaid at 50 years: perspectives of beneficiaries, health care professionals and institutions, and policy makers. JAMA. 2015;314(4):384-95. https://doi. org/10.1001/jama.2015.7811

15. Rice T, Unruh LY, Ginneken E, Rosenau P, Barnes AJ. Universal coverage reforms in the USA: fom Obamacare through Trump. Health Policy. 2018;122(7):698-702. https://doi.org/10.1016/j. healthpol.2018.05.007 
16. Sen A. Equality of What? In: McMurrin S, editor. Tanner lectures on human values. Vol. 1. Cambridge (UK): Cambridge University Press; 1980.

17. Sen A. Inequality reexamined. Oxford (UK): Clarendon Press; 1992.

18. Nussbaum MC. Frontiers of justice: disability, nationality, species membership. Cambridge, MA: Harvard University Press; 2009.

19. Nussbaum MC. Creating capabilities: the human development approach. Cambridge, MA: Harvard University Press; 2011.

20. Comisión para Reducir las Desigualdades Sociales en Salud en España. Propuesta de políticas e intervenciones para reducir las desigualdades sociales en salud en España. Gac Sanit. 2012;26(2):182-9. https://doi.org/10.1016/j.gaceta.2011.07.024

21. Marmot M, Allen J, Bell R, Bloomer E, Goldblatt P; Consortium for the European Review of Social Determinants of Health and the Health Divide. WHO European review of social determinants of health and the health divide. Lancet. 2012;380(9846):1011-29. https://doi. org/10.1016/S0140-6736(12)61228-8

22. Puyol A. Ética, equidad y determinantes sociales de la salud. Gac Sanit. 2012;26(2):178-81. https://doi.org/10.1016/j.gaceta.2011.08.007

23. Urbanos-Garrido R. La desigualdad en el acceso a las prestaciones sanitarias. Propuestas para lograr la equidad. Gac Sanit. 2016;30 Supl 1:25-30. https://doi.org/10.1016/j. gaceta.2016.01.012

24. Glaser BG, Strauss AL. The discovery of grounded theory: strategies for qualitative research. New York: Aldine de Gruyter; 1967.

25. Bardin L. Análisis de contenido. 2. ed. Madrid: Ediciones Akal; 1996.

26. Mayring P. Qualitative content analysis. Forum Qual Soc Res. 2000 [citado 17 nov 2019];1(2):20. Disponible en: http://www.qualitative-research.net/index.php/fqs/article/ view/1089/2385

27. Silva SAG, Duarte RG, Castro JM. Transfer of knowledge in international cooperation: the Farmanguinhos-SMM case. Rev Saude Publica. 2017;51:103. https://doi.org/10.11606/s15188787.2017051006249

28. Whitehead M, Dahlgren G. Levelling up (part 1): a discussion paper on concepts and principles for tackling social inequities in health. Copenhagen (DK): WHO Regional Office for Europe; 2006 [citado 14 dec 2019]. (Studies on social and economic determinants of population health; $\mathrm{n}^{\circ}$.2). Disponible en: https://apps.who.int/iris/handle/10665/107790

29. Borrell C, Peiró R, Ramón N, Pasarín MI, Colomer C, Zafra E, et al. Desigualdades socioeconómicas y planes de salud en las comunidades autónomas del Estado español. Gac Sanit. 2005;19(4):277-85. https://doi.org/10.1157/13078025

30. Briones-Vozmediano E, Vives-Cases C, Peiró-Pérez R. Gender sensitivity in national health plans in Latin America and the European Union. Health Policy. 2012;106(1):88-96. https://doi. org/10.1016/j.healthpol.2012.03.001

31. Fiuza Pérez MD, Aguiar Rodríguez JF, Monzón Batista N. Una década de reflexión sobre los planes de salud en España. Informe SESPAS 2010. Gac Sanit. 2010;24 Supl 1:37-41. https://doi. org/10.1016/j.gaceta.2010.10.005

Financiamiento: Esta publicación se enmarca en las actividades del proyecto de investigación "Brecha digital e inhibidores en la implementación del e-Gobierno: Especial impacto en el ámbito de la salud” (CSO2014-53014-R) (2015-2018), financiado por el Ministerio de Economía y Competitividad (Programa Estatal de Investigación, Desarrollo e Innovación Orientada a los Retos de la Sociedad, Convocatoria 2014). Este hecho no supone la existencia de ningún tipo de conflicto de interés ni por dicha financiación ni por otros motivos.

Contribución de los Autores: Concepción y planificación del estudio: BBB, RBL, ACR, AFD. Recolección, análisis e interpretación de los datos: BBB, RBL, ACR, AFD. Elaboración o revisión crítica del manuscrito: BBB, RBL, ACR, AFD. Aprobación de la versión final: BBB, RBL, ACR, AFD.

Conflicto de Intereses: Los autores no tienen conflicto de interés. 